\title{
Real-Time Control of Human Coagulation
}

\author{
Joseph G. Makin* and Srini Narayanan \\ EECS Department, University of California, Berkeley, USA \\ International Computer Science Institute, 1947 Center Street, Berkeley, CA 94704, USA
}

\begin{abstract}
In previous work [1] we showed that the dynamics of human blood clotting could be fruitfully modeled and simulated as a hybrid system (HS), i.e. one with interacting continuous and discrete parts. Here we show that, although a complete analysis of the hybrid system is (computationally) infeasible, analysis and control techniques can indeed be applied to a large, critical subsystem (corresponding more or less to the model of [2]), a set of about 80 ordinary differential equations. We outline the theory behind the control techniques and then demonstrate in a series of simulations their application to control of pathological blood clotting, both hypercoagulatory (Factor-V Leiden) and hypocoagulatory (hæmophilia A). In particular, we simulate steering during a clotting event of the crucial blood-protein thrombin, via controlled injection of recombinant factor VIII (for hæmophilia) or the anti-coagulant heparin (for FV Leiden). It remains to remedy the shortcomings of this control technique, and to extend it to the remainder of the hybrid system of [1]; methods for these are proposed, and addressed in a subsequent paper.
\end{abstract}

\section{Author Summary}

Human blood clotting is a complicated and not fully understood biological process, which both makes it interesting to researchers and renders treatment of its disorders difficult. Indeed, current treatments make no attempt to manipulate coagulation in real time; rather, periodic (on the order of one day to one week) measurements and interventions are made with the aim of keeping certain known risk factors within safe ranges. This limitation is a consequence of two facts: first, the state of current sensor technology, which precludes real-time measurements of blood proteins (and hence real-time feedback control); and second, the lack of theoretical techniques for such control. This paper address the second of these limitations by applying techniques from computer science and from mathematical control theory and demonstrating their validity-and limitations - both mathematically and in a series of simulations. By providing part of the solution to the theoretical problem of controlling blood clotting,

*Author to whom correspondence should be addressed (makin@icsi.berkeley.edu) 
we expect to provide an impetus for the development of the relevant sensor technologies.

\section{Introduction}

Previously [1], we proposed a hybrid-system approach to human blood clotting, constructed a model in that spirit, and performed a series of simulations of the coagulation cascade in normal and pathological cases. Hybrid systems are heterogenous in virtue of containing both continuous and discrete parts (time or state space). The rationale behind the approach was that blood clotting, like other complicated biological processes, necessarily involves both continous-time, continuous-state dynamics - as in the ordinary differential equations (ODEs) arising from the the chemical equations of protein kinetics - as well as discrete events (like thresholds) and discrete states (e.g. presence/absence of calcium). In particular, inclusion of discrete elements allows us to capture qualitative information, and consequently to model the entire blood-clotting process. We stress that the complete clotting cascade cannot be rendered as a set of ODEs, and that such models as do exist - e.g. [3], [4], [2], [5], [6], [7], [8], and [9] - treat only a subset of the coagulation cascade.

The simulations demonstrated the utility of the model for certain purposes (prediction, theoretical investigation, sensitivity analysis), but ultimately we should like to use it as a basis for the real-time control of blood-clotting. Now, the standard technique for control of hybrid systems is numerical solution of a set of partial differential equations - the Hamilton-Jacobi equations - which for systems of dimension greater than about five is computationally infeasible (a consequence of the curse of dimensionality) [10]. Unfortunately, our system has upwards of 100 state variables, so this technique is patently unworkable.

The approach taken here is to consider only a subsystem of the model, a set of nonlinear ODEs which were originally drawn largely from [2]. In fact (though we do not show it here), the model can be decomposed into this (purely continuous) subsystem and two hybrid systems, the three interacting only through two state variables. In a subsequent paper we will show how this decomposition can be exploited, along with control of the ODEs, to control clot formation in the complete model; in the present study we content ourselves with deriving and simulating the control techniques for the system of ODEs alone. Specifically, the control task is to steer the concentration of thrombin (activated blood factor II) along a desired trajectory during a clotting event, by controlled rate of injection of an exogenous pharmaceutical (e.g. heparin). We demonstrate two different techniques to effect this control: the first, following [11], using nonlinear feedback and a change of variables to partially linearize the ODEs, and then controlling the linearized, single-input/single-output (SISO) system using standard methods; and the second based on step-input control. In the simulations, the input is either the anti-coagulant heparin or (recombinant) factor VIII, and the output is thrombin, the most important enzyme product of the coagulation cascade (see below). 
We present the theory of the control techniques first, along with some preparatory results on alternative approaches, before presenting a series of simulations. We then simulate the clotting process in a patient with the procoagulatory disorder Factor-V Leiden and in a patient with moderate hæmophilia $\mathrm{A}$, and then repeat the simulation under application of the control techniques. Finally, we discuss both the relevance of this control task to the overall task of controlling blood clotting, as well as implementation issues, and then propose an alternative technique to remedy some of the defects of the present approach.

\section{Theory}

The model to be controlled is a system of about 80 coupled, nonlinear ODEs, of the most general form,

$$
\dot{\mathbf{x}}=f(\mathbf{x}, u)
$$

where $u$ is the (single) control variable (say, an anti- or pro-coagulant). The control task is to force one of the state variables (thrombin) to track a desired trajectory. In fact, the ODEs can be expressed, as we shall see, in a less general form; and the approach described in this paper will be to exploit some of the peculiarities of the system which distinguish it from the most general case.

Now, control of linear systems is comparatively easy, so a standard approach is to design the controller around a linear approximation to the true system, found by considering only the first term of the Taylor expansion, the Jacobian $\frac{\partial f}{\partial \mathbf{x}}\left(\mathbf{x}_{\mathbf{0}}\right)$, near an equilibrium point $\mathbf{x}_{\mathbf{0}}$. Unfortunately, the Jacobian in our model is singular, so (by the Hartman-Grobman theorem) the linearization is not guaranteed to approximate the true system.

Alternatively, the system may be exactly linearized (as opposed to merely approximated by a linear system) by choosing the appropriate nonlinear feedback $u=\psi(h(\mathbf{x}))$ and looking at the system through a change of variables $\xi=\Phi(\mathbf{x})$ :

$$
\begin{aligned}
\dot{\xi} & =A \xi+b v, \\
y & =\xi_{1},
\end{aligned}
$$

where in fact $(A, b)$ are in controllable canonical form, so the system is completely controllable. Here $h(\mathbf{x})$ is an output function which reflects our observations of the state variables, and $v$ is a synthetic input related to the true input $u$ by a known function. From this perspective we can ask whether or not, given the dynamics in (1), there exists an output funtion rich enough to support the linearization. The answer is particularly straightforward if, as in our case, the ODEs are affine in the control, i.e. can be written as:

$$
\dot{\mathbf{x}}=f(\mathbf{x})+g(\mathbf{x}) u,
$$

in which case necessary and sufficient conditions for the linearization can be given in the form of conditions on the vector fields $f(\mathbf{x})$ and $g(\mathbf{x})$ and iterated Lie brackets thereof. Specifically, the matrix of vector fields

$$
\left[g(\mathbf{x}), a d_{f} g(\mathbf{x}), \ldots, a d_{f}^{n-2} g(\mathbf{x}), a d_{f}^{n-1} g(\mathbf{x})\right],
$$


often referred to as the strong-accessibility distribution, must have full rank $(n)$ in the region of interest; and the set of vector fields

$$
g(\mathbf{x}), a d_{f} g(\mathbf{x}), \ldots, a d_{f}^{n-3} g(\mathbf{x}), a d_{f}^{n-2} g(\mathbf{x})
$$

must be involutive* in the region. Here, by recursive definition,

$$
\begin{array}{lll}
a d_{f}^{k} g:=\left[f, a d_{f}^{k-1} g\right], & k>0 \\
a d_{f}^{k} g:=g, & k=0 .
\end{array}
$$

If these conditions are not met and full-state linearization is not possible, one may attempt to partially linearize the system. Here, the linearization is carried out with respect to some given output function, which will admit the formulation of some number $q$ of new state variables with linear dynamics. Of course, if full-state linearization is not possible, then $q$, called the relative degree of the affine-control system, is strictly less than $n$, the dimension of the state space.

Consider again the differential equations of our system as the affine-control system of Eq. (3), where this time $h(\mathbf{x})=y$, the variable we wish to control. Differentiating the output with respect to time yields:

$$
\begin{aligned}
\dot{y} & =\frac{\partial h}{\partial \mathbf{x}} \dot{x} \\
& =\frac{\partial h}{\partial \mathbf{x}} f(\mathbf{x})+\frac{\partial h}{\partial \mathbf{x}} g(\mathbf{x}) u \\
& =L_{f} h(\mathbf{x})+L_{g} h(\mathbf{x}) u
\end{aligned}
$$

where $L_{\eta} \lambda(\mathbf{x}):=\frac{\partial \lambda}{\partial \mathbf{x}} \eta(\mathbf{x})$, the Lie derivative of the function $\lambda$ along the vector field given by $\eta$. Now if $L_{g} h(\mathbf{x})$ is nonzero, then the system is said to have a strict relative degree of one, and we can force it to track a desired trajectory $y_{d}$ by choosing:

$$
u_{d}=\frac{1}{L_{g} h(\mathbf{x})}\left(\dot{y}_{d}-L_{f} h(\mathbf{x})\right)
$$

and making sure that the initial conditions match $\left(y(0)=y_{d}(0), \dot{y}(0)=\dot{y}_{d}(0)\right)$. Changing variables according to $\xi=\Phi(\mathbf{x}):=h(\mathbf{x})$ and defining for the nonce $\dot{y}_{d}=: v$, a synthetic input, we see a one-dimensional linear system $\dot{\xi}=0 \cdot \xi+v$ and an $(n-1)$-dimensional nonlinear system $\dot{\eta}=\lambda(\xi, \eta)$. Notice that the linear system is decoupled from the nonlinear one, in the sense that $\dot{\xi}$ is not a function of $\eta$.

If, on the other hand, $L_{g} h(\mathbf{x})$ is zero, then we differentiate $y$ a second time to get:

$$
\ddot{y}=L_{f}^{2} h(\mathbf{x})+L_{g} L_{f} h(\mathbf{x}) u,
$$

*A set of vector fields is involutive if the Lie bracket of any two of those vector fields is within the span of the original set, where the Lie bracket $[f, g]$ of two vector fields is defined as $\frac{\partial g}{\partial \mathbf{x}} f-\frac{\partial f}{\partial \mathbf{x}} g$. 
and, if $L_{g} L_{f} h(\mathbf{x}) \neq 0$ (i.e. the system has strict relative degree two), choose:

$$
u_{d}=\frac{1}{L_{g} L_{f} h(\mathbf{x})}\left(\ddot{y}_{d}-L_{f}^{2} h(\mathbf{x})\right)
$$

this time making sure that $\ddot{y}(0)=\ddot{y}_{d}(0)$ as well. Choosing $\xi=\Phi(\mathbf{x}):=$ $\left[h(\mathbf{x}), L_{f} h(\mathbf{x})\right]^{T}$ again yields a linear system (this time two-dimensional) which is decoupled from the remaining $(n-1)$-dimensional nonlinear system.

In general, for a system of strict relative degree $q$, we choose

$$
u_{d}=\frac{1}{L_{g} L_{f}^{q-1} h(\mathbf{x})}\left(y_{d}^{(q)}-L_{f}^{q} h(\mathbf{x})\right),
$$

and set all the initial conditions $y_{d}(0), \dot{y}_{d}(0), \ldots, y_{d}^{(q)}(0)$ appropriately. Note that, if $q=n$, the dimension of the state space, then

$$
\Phi(\mathbf{x}):=\left[h(\mathbf{x}), L_{f} h(\mathbf{x}), \ldots, L_{f}^{n-1} h(\mathbf{x})\right]^{T}
$$

is a valid change of coordinates (locally diffeomorphic), which in fact puts the system into controllable canonical form-i.e., (3) is fully linearizable by state feedback.

\section{Application to the model}

\subsection{Feedback Linearization}

Full-state linearization as lately described faces a significant issue when applied to the present model. Although verifying the two conditions associated with (4) and (5) is mathematically straightforward, it is computationally intensive. Computing the vector fields requires the computation of two Jacobians for every Lie derivative, and about 80 Lie derivatives. Calculating each Jacobian itself entails about $80^{2}$ derivatives of polynomials. Even neglecting the multiplication and addition operations, this brings the total to nearly a million computations - each of which is a (symbolic) derivative of a polynomial, increasing the total number of computations yet more. And most unfortunately of all, each derivative (at least potentially) generates more variables, via the chain rule of differentiation, so the polynomials have increasingly more terms in later vector fields.

Apart from computational difficulties, it remains to construct the output function, $h(\mathbf{x})$. No mechanical procedure for this construction exists.

In fact, we can circumvent the computational obstacles: [12] has shown that, in the case of reaction systems like the present one, the strong-accessibility rank is bounded from above by the rank of a matrix (the "accessibility matrix") which is independent of $x$, and is computed very simply. The existence of such a matrix depends once again on being able to express the governing ODEs in an even more specific form. In particular, Eq. 3 can be written

$$
\dot{\mathbf{x}}=C r(\mathbf{x})+b u,
$$


where each element in the vector $r(\mathbf{x})$ is a monomial corresponding to one of the chemical reactions of the system (i.e one of the arrows in the chemical formulae); and $b$ is a constant vector, i.e. does not depend on the state. The present case is a special case even of the one discussed in [12]; for this system, the accessibility matrix is the augmented matrix $M:=[b, C]$, and it can easily be shown that

$$
\forall \mathbf{x}, \quad r k(M) \geq r k\left(\left[g(\mathbf{x}), a d_{f} g(\mathbf{x}), \ldots, a d_{f}^{n-2} g(\mathbf{x}), a d_{f}^{n-1} g(\mathbf{x})\right]\right) .
$$

Crucially, the rank of $M$ is easy to compute, and we avoid crunching through the iterated Lie derivatives of the right-hand side.

Using heparin as the single input, $r k(M)<n$, the dimension of the state space, so the system is not fully linearizable by state feedback; i.e. there is no single-variable output function $h(\mathbf{x})$ that can be constructed to support such feedback. In fact, chemical-reaction systems will almost always have fewer reactions (arrows) than state variables (chemicals), so $C$ will almost always have fewer columns than rows (i.e. $n$ ). Thus even if $C$ has full rank, the strongaccessibility rank will generally be less than $n$.

So the system is not completely controllable. However, there is reason to believe that clotting can be controlled just by controlling the concentration of thrombin (activated factor II): Clotting occurs downstream in the coagulation cascade of the ODEs of this study, and interacts with them only via thrombin. (See e.g. the hybrid-system model of [1].) Thus we attempt to partially linearize the system of Eq. 3 with thrombin the output $y=h(\mathbf{x})$. Indeed, using heparin as the control variable and augmenting the system with the appropriate terms from the heparin chemical reactions, the strict relative degree of the system is two. ${ }^{\dagger}$ Alternatively, using Factor VIII as the input yields a strict relative degree of three.

Now, in practice, small numerical discrepencies (arising e.g. from a mismatch between the plant and the model, or from numerical approximations) are eliminated by including in the controller a proportional and an integral term [13] This is accomplished by replacing the $y_{d}^{(q)}$ term in Eq. 10 with the synthetic input

$$
v(t)=y_{d}^{(q)}(t)+K_{p}\left(y_{d}(t)-y(t)\right)+\frac{K_{i}}{t} \int_{0}^{t}\left(y_{d}(\tau)-y(\tau)\right) d \tau .
$$

The gain terms, $K_{p}$ and $K_{i}$, are adjusted by hand.

Finally, practical application will also require discretization of the controller. The sampling rate of the controller affects the controllability of the system, so under a sufficiently low rate the theory lately outlined will fail to achieve

\footnotetext{
${ }^{\dagger}$ Lacking complete controllability, is there something the system has gained? I.e., is there a trade-off in the area? An affirmative answer can be given if one considers the computational complexity: In order to compute the feedback law, we need only calculate (two) twice-iterated Lie brackets; whereas if the system were completely controllable, the two vector fields would require the calculation of $n$-iterated Lie brackets. In a system as complicated as ours, this would be a serious problem, even given a simple output function. Additionally, we need only specify two, rather than $n$, initial conditions. This question is even more relevant when an output function which will provide strict relative degree $n$ is available.
} 
exact output tracking. We demonstrate a working discrete controller in the simulations below.

\subsection{Step-Input Control}

A much simpler control technique was also applied; the rationale for it is discussed below. This strategy is predicated on the assumption that a single step input might suffice to force the system to track the desired thrombin trajectory "reasonably well." More precisely, the assumption is that the step input that matches both the desired peak concentration of thrombin and the occurrence of this peak will result in a thrombin trajectory that deviates very little over its entirety from its desired counterpart. Therefore this technique was implemented by performing a parameter search over repeated trials for that step input which would minimize the peak-concentration and peak-time discrepencies. Thus the input $u$ was modified on successive trials by modification according to:

$$
u=u+\alpha\left(\max _{t}\{y(t)\}-\max _{t}\left\{y_{d}(t)\right\}\right)+\beta\left(\underset{t}{\operatorname{argmax}}\{y(t)\}-\underset{t}{\operatorname{argmax}}\left\{y_{d}(t)\right\}\right),
$$

where $\alpha$ and $\beta$ - positive for anti-coagulant inputs and negative for pro-coagulantsadjust the relative contribution of each term, and were adjusted by hand.

\section{Methods}

The ODEs for the non-intervention case are derived from the chemical equations in [2], from which also the rate constants have been taken. They are reproduced here in Table 1. The blood factors are referred to by their Roman-numeral designations, a lowercase "a" denoting the activated form. Other abbreviations inlude: mIIa for meizothrombin, LBS for the concentration of lipid binding sites, PS for protein S, PC for protein C, APC for its activated form, TFPI for tissue-factor-pathway inhibitor, Tm for thrombomodulin, AT for antithrombin, and TF for tissue factor. A subscripted "i" indicates the inactivated form of an enzyme.

The form of the heparin equations comes from [14] and appear in Table 2. The abbreviated proteins are heparin, thrombin (activated factor II), and antithrombin; (IIa-AT) is a stable complex which can no longer dissociate into inhibitor and protease.

Such are the forms of the heparin reactions; however, to these authors' knowledge, the exact values of the rate constants under physiological conditions have never been determined. The following expedient was therefore adopted: on and off rates were set to intermediate values within the biologically normal ranges for enzyme-substrate reactions (see for example Table 4.4 in [15], but also [2]): $k_{\text {on }}$ rates were set at $0.1 \mathrm{~s}^{-1} \mathrm{nM}^{-1}$ and $k_{\text {off }}$ rates at $10 \mathrm{~s}^{-1}$. This approximation does not vitiate the theoretical apparatus, but it does have numerical consequences which will be considered in the discussion below. Heparin is also thought to facilitate the inactivation of Factor $\mathrm{Xa}$, but for simplicity these interactions have been neglected. 
Table 3 lists all the non-zero initial concentrations. The concentration of lipid binding sites and the intial activating amount of tissue factor were chosen to match [16], as in [2]. All other concentrations are normal physiological values.

All simulations were performed in MATLAB using the stiff ODE solver ode15s. Computation of the strict relative degree of the system were also performed in MatLaB, using the symbolic toolbox.

\section{Results}

We first simulate coagulation in a patient with the hypercoagulatory disorder Factor-V Leiden, with and without intervention by the anti-coagulant heparin, as well as normal (nonpathological) clotting. Initiation of the clotting event is assumed to take place via the intrinsic pathway and is therefore modeled by initializing tissue factor at a concentration of $5 \mathrm{pM}$ (following [2]).

Factor-V Leiden renders the blood protein factor Va (a pro-coagulant) resistant to inactivation by the activated-protein- $\mathrm{C} /$ protein-S complex. It was modeled simply by eliminating this chemical reaction (thus removing the corresponding monomials from the differential equations).

Figure 1 shows the thrombin profile during normal clotting (dark blue), clotting in a "patient" with Factor-V Leiden (light blue), and that clotting in the same patient but with the feedback-linearizing controller (green). Exact output tracking has been achieved; however, several defects are immediately obvious. First, the controller operates continuously (i.e. updating as often as the numerical simulation of the ODEs does), whereas any practical controller must be digital. Second, the controller is required to make a large excursion about 215 seconds into the simulation, a consequence of the nonlinearities in the control law (i.e. the Lie derivatives in Eq. 10. The magnitude of the excursion impugns the accuracy of the numerical simulation of the ODEs in that region. Third, the rate of heparin input should be limited; certainly, it cannot be negative, since this implies withdrawal of heparin from the site of the injury.

We address the final defect first. Note, however, that the theory outlined above does not guarantee the success of any these remedies. As lately noted, the input cannot be a negative number, nor can it be much higher than a singledigit nanomolarity. ${ }^{\ddagger}$ We impose an upper bound of $2 \mathrm{nM} / \mathrm{s}$ and a lower bound of zero by "squashing" inputs outside this range, i.e. setting any $u$ calcuted from Eq. 10 which exceeds one of these bounds to the value of the bound. The result appears in Fig. 2.

The controller gains were optimized to yield the best match to the desired trajectory, but clearly naïvely constraining the outputs significantly degrades the tracking. The results for the digital controller were similar: Discretizing at a $2 \mathrm{~Hz}$ sampling rate gives the best achievable tracking, but this is still defective (Fig. 3).

\footnotetext{
${ }^{\ddagger}$ Continuous intravenous infusions over a 24 -hour period total between 20,000 and 40,000 IU (International units), which comes to about $10.29 \mathrm{pM} / \mathrm{s}$.
} 


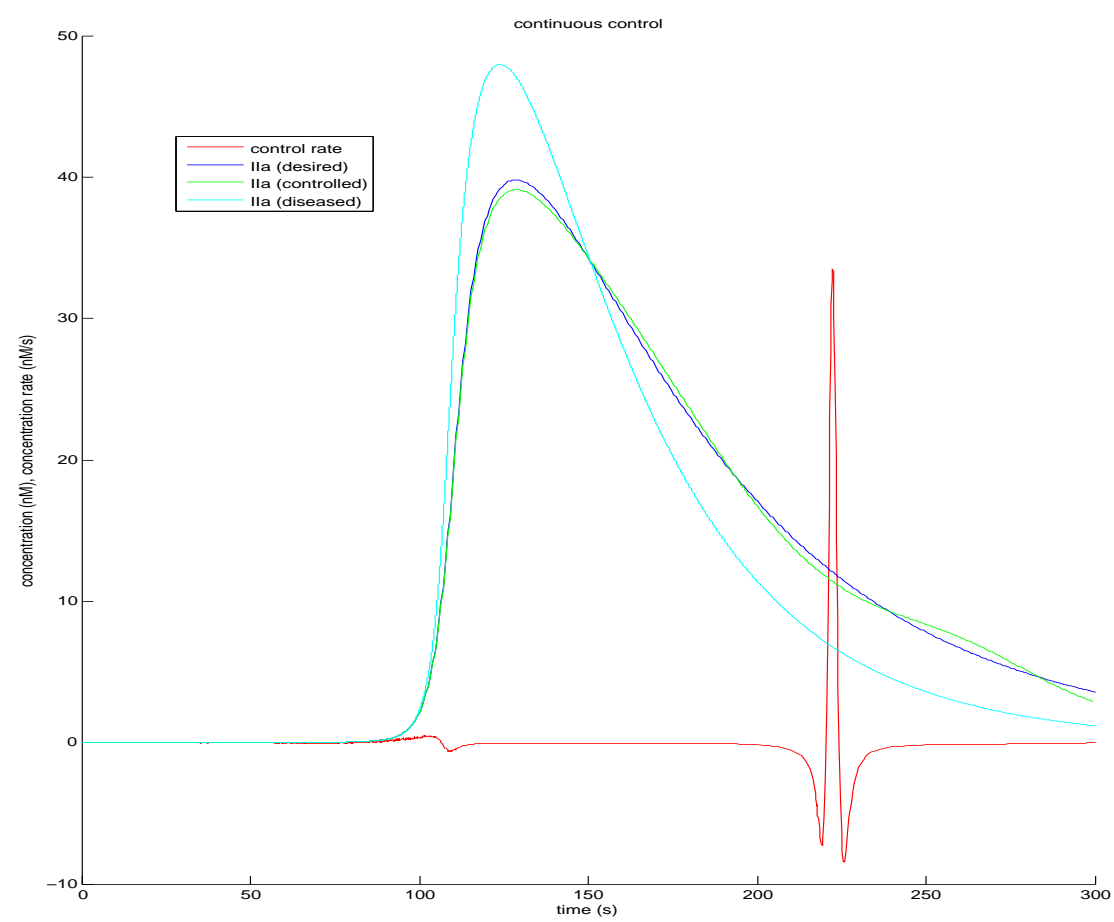

Figure 1: Simulated control of thrombin concentration during a clotting event in a patient with Factor-V Leiden. The controller gains were set to $0.005\left(K_{p}\right)$ and $0\left(K_{i}\right)$ and the controller updates continuously

Whether the controlled trajectories of Figures 2 and 3 present a hypocoagulatory risk is something of an open question: Rapid product formation "downstream" in the cascade - i.e. the formation of fibrin from fibrinogen and activation of Factor XIII - requires concentrations of thrombin less than $2 \mathrm{nM}$ [17], so the low peak may not critically affect clotting. (Nevertheless, it may, and so we propose a more sophisticated approach in section 8 below.)

How well can we do with our simple, step controller? Better, in fact, than with the previous technique. Choosing $\alpha=5 \times 10^{-4}$ and $\beta=5 \times 10^{-5}$ in Eq. 15 , the input will settle on $u=9.5 \mathrm{pM} / \mathrm{s}$, at which rate the peak concentration and its occurrence can be very nearly matched (Fig. 4).

Finally, we consider moderate hæmophilia A, at which native levels of the zymogen Factor VIII are about $2.5 \%$ of normal levels. Applying the step-input technique (but using $\alpha=1 \times 10^{-4}$ and $\beta=1 \times 10^{-5}$ ) again yields remarkably faithful tracking, as seen in Fig. 5, this time with an input of Factor VIII at 6.7 $\mathrm{pM} / \mathrm{s}$. 


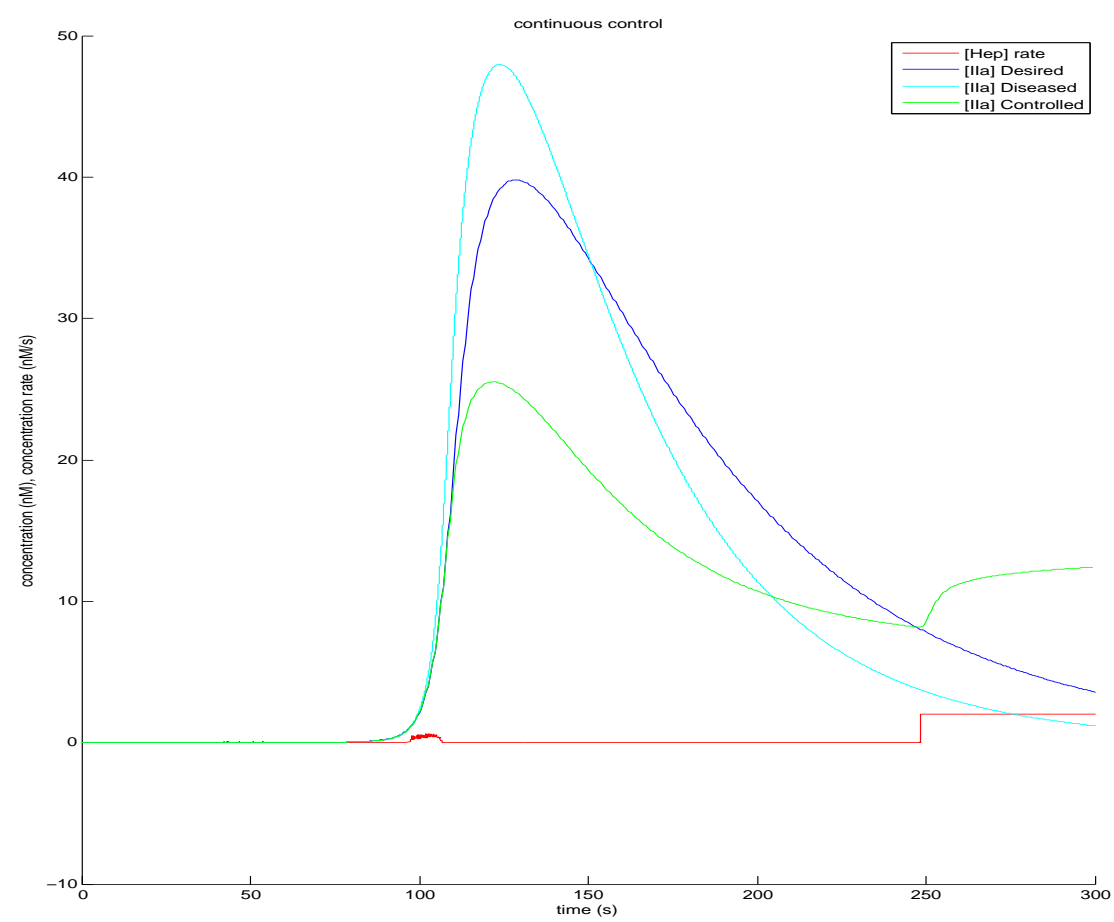

Figure 2: Simulated control of thrombin concentration during a clotting event in a patient with Factor-V Leiden, again continuously sampling. The controller gains were set to $0.1\left(K_{p}\right)$ and $0\left(K_{i}\right)$ and the input was constrained in the range between 0 and $2 \mathrm{nM} / \mathrm{s}$.

\section{Discussion}

We have shown via an algebraic criterion that our system of ODEs is not fullstate linearizable. As a matter of fact, we can say more. It follows from the method of transforming chemical equations into ODEs that every reversible reaction will introduce two identical columns into $C$ [12]. Thus, while the rank of $C$ is obviously upper-bounded by the number of unidirectional reactions (i.e. the number of arrows in all our chemical equations) since this is the number of columns in $C$, it is also upper-bounded by the (generally smaller) number of unior bidirectional reactions (i.e. counting each pair of arrows as just one reaction; henceforth simply called "reactions"). If this number falls short of the dimension of the state space, then full-state linearization requires the deficit to be covered by the control vector fields - which, in the case of a single-input, can provide additional rank of at most one. And indeed, the number of reactions in our 


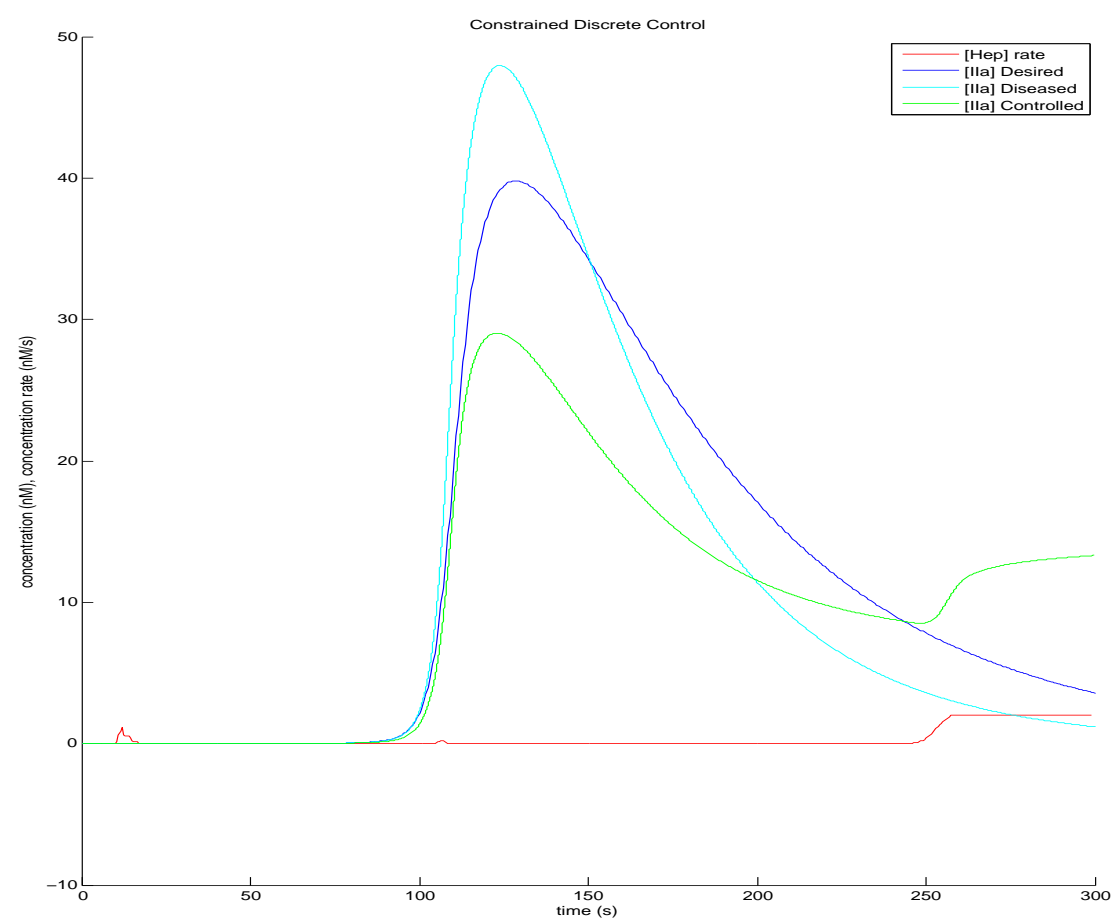

Figure 3: Simulated control of thrombin concentration during a clotting event in a patient with Factor-V Leiden, this time using a discrete controller sampling every $0.5 \mathrm{~s}$. The controller gains were set to $0.1\left(K_{p}\right)$ and $0\left(K_{i}\right)$ and the input was constrained in the range between 0 and $2 \mathrm{nM} / \mathrm{s}$.

system falls well short of $n$. So in chemical systems of mass-action kinetics with no outflow, a single output, and a single input, a necessary (though insufficient) condition for full-state linearization is that there be at least as many reactions as state variables.

Again considering the present system, we find that without the heparin reactions there are 66 reactions and 73 state variables. Now, we are obviously interested in pharmaceutical interventions in addition to heparin (a whole host of pro-coagulants, for instance). But it can now be claimed that in order to even stand a chance of fully linearizing the system with one of these interventions as the (sole) input, the drug must interact with the system via at least six more reactions than the number of variables that these reactions introduce. (Recall that each reaction will generally introduce more state variables, either as compounds of other chemicals or as "activated," and hence new, factors.)

Finally on the topic of full state linearization, and interestingly, it can be 


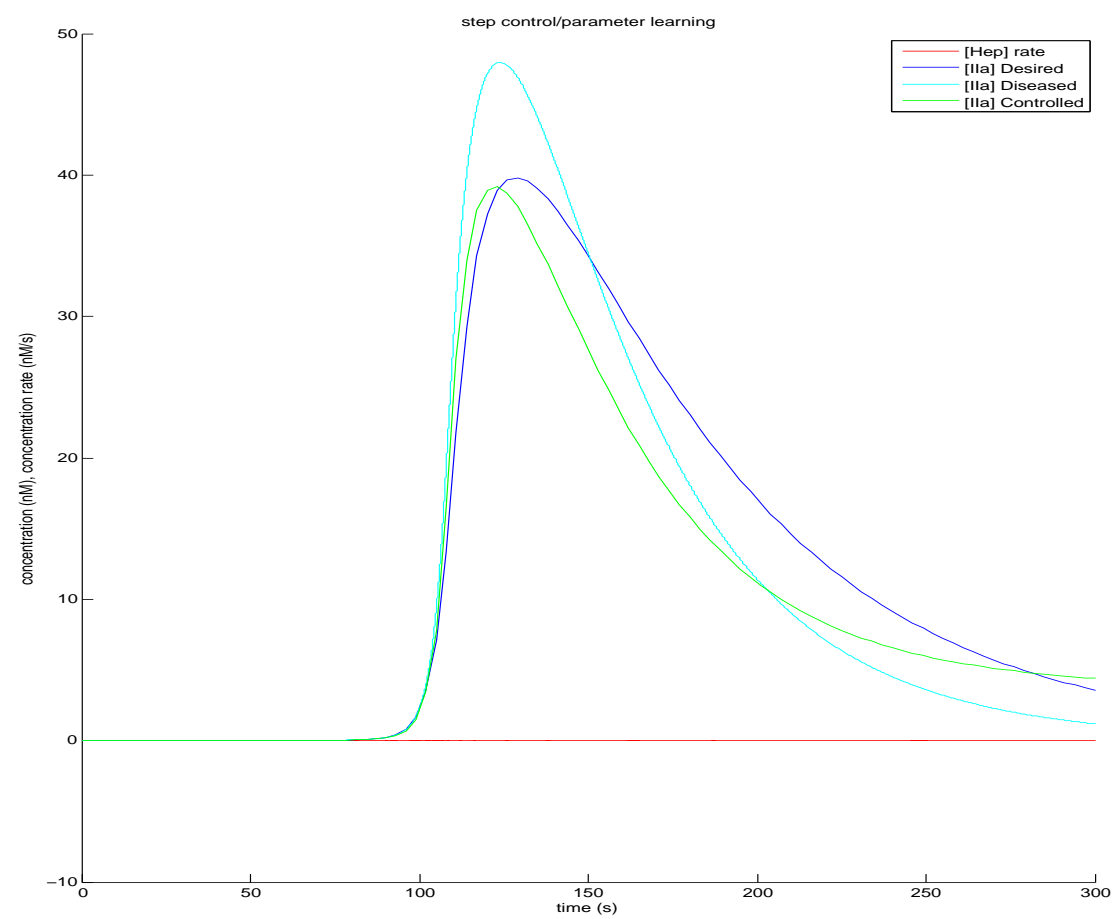

Figure 4: Control of thrombin concentration in a patient with FV Leiden by a single step input at time $t=0$. The input is $9.5 \mathrm{pM} / \mathrm{s}$.

shown that outflow of the blood factors - in particular, heterogeneous outflowcan increase the rank of the accessibility matrix, and hence of the strongaccessibility distribution [12]. For simplicity, the model as it stands neglects outflow, but in fact in vivo coagulation will perforce have some flow (both in and out) of clotting factors, and if it be not negligible over the time scale of interest, may provide for greater controllability of the system.

But how precise does our control need to be, after all? The answer is not known, but there is some suggestion that the required amount of thrombin for clotting is much less than the actual peak concentration: Brummel et al. have demonstrated in an in vitro study that less than $2 \mathrm{nM}$ of thrombin is required for rapid product formation downstream in the cascade [17]. Certainly this suggests that step inputs suffice to steer hæmophilia-A and Factor-V-Leiden patients through safe clotting, given the qualitative matches of Figs. 4 and 5. In case these matches are not sufficient, however, we propose in the following section a model-predictive approach to remedy the defects of the feedback-linearizing controller. 


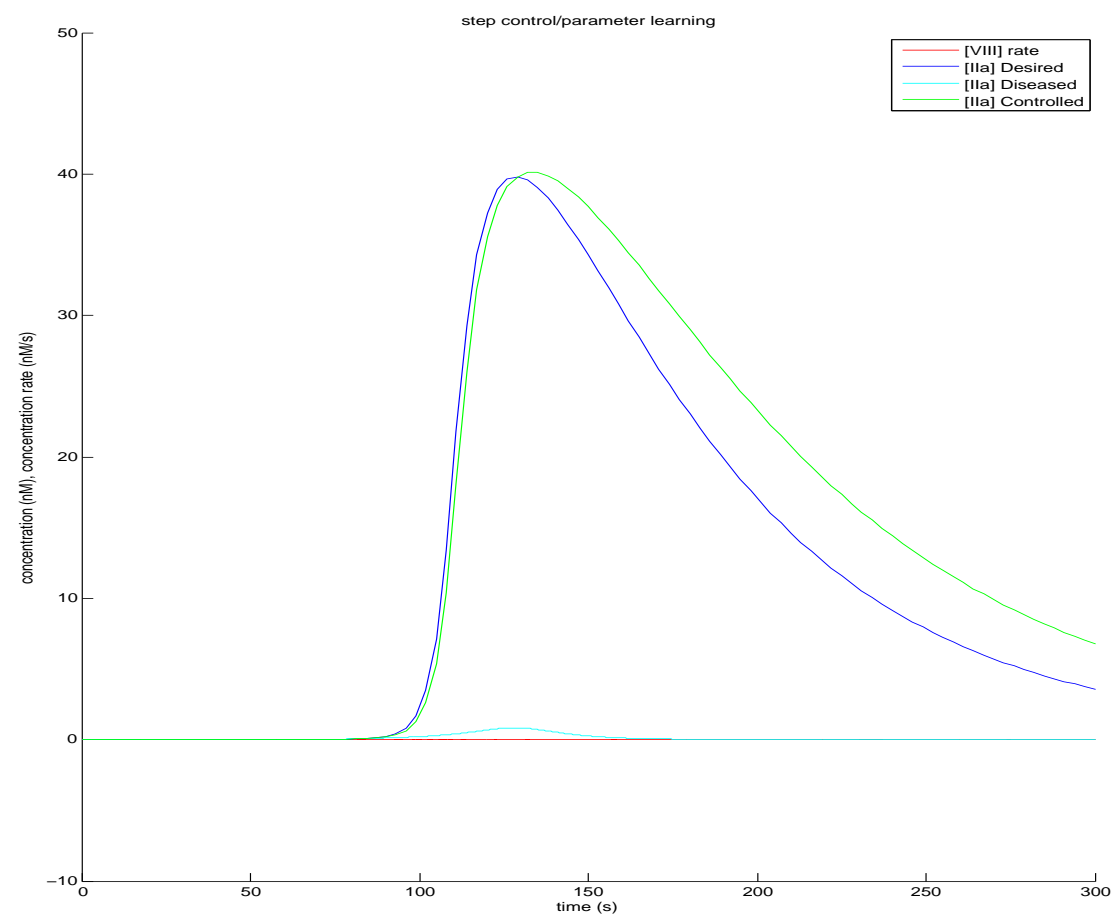

Figure 5: Control of thrombin concentration in a hæmophiliac using a single step input at time $t=0$. The input is $6.7 \mathrm{pM} / \mathrm{s}$.

However, the step controllers have another enormous advantage over the feedback-linearizing control: they require no sampling, only initiation at the inception of a clotting event. There is not currently a method for measuring blood-protein concentrations in real time - certainly not at the required $2 \mathrm{~Hz}$ sampling rate. We interpret our results on feedback linearization, then, as showing that if indeed a greater degree of accuracy is required for thrombin tracking, then it would be fruitful to devise the appropriate sensors for realtime sampling, since they would make possible this suitable technique. Now, naïvely constraining the input vitiates the accuracy of this technique (Figs. 2 and 3), hence the need for the model-predictive controller.

Two more model assumptions require elaboration. First, both controllers show sensitivity to the choice of rate constants; recall that the unknown heparin on and off rates were chosen in a reasonable physiological range ( 55$)$. Specifically, increasing the disparity between on and off rates makes control more difficult, even in the case of continuous control and no constraints on the input. This suggests that the difficulty is numerical rather than theoretical. In 
particular, if the off rates are three or more orders of magnitude greater than the on rates, then the unconstrained continuous controller is required to make greater excusions like the one seen in Fig. 1, jeopardizing the numerical stability of the ODE solver. And of course, these excursions would be prevented by any serious input constraints, so in a realistic scenario this controller might be unsuitable. Similarly, the step-input controller under such on and off rates can succeed in matching the thrombin peak only by greatly distorting the subsequent thrombin trajectory (not shown), so its usefulness in treating Factor- $\mathrm{V}$ Leiden is limited, too, by the actual values of the rate constants. Inasamuch as the rate constants for the non-heparin reactions are fairly well established [2], however, the step controller's utility in treating hæmophilia A is unaffected by these considerations.

Finally, these simulations were carried out under an assumption of locality: the supply of unactivated (zymogen) blood factors was treated as limited. This choice was made to conform to the model of [2] as well as to the in vitro results of [16]. In reality, however, zymogens are replenished by circulating blood, and activated proteins are similarly removed. On the other hand, the limited amount of lipids also restricts the amount of each zymogen which can ever take part in the reaction, since nearly all of the clotting reactions take place on a phospholipid surface. Congruently, simulations (not shown) indicate that even if the zymogens are modeled as inexhaustible, the thrombin curve remains qualitatively the same. We proceeded then with the locality assumption, in order to obviate the complexities of an added circulation model.

\section{Future Work}

The significance of both the controllers presented in this paper for clinical application would be greatly improved by various improvements to the model. First, as lately noted, the rate constants for heparin interactions with the clotting process are not known; if a precise controller is to be constructed along the lines proposed here, these must be determined. Second, a circulation model would dispense with the somewhat dubious locality assumption, as well as possibly afford more powerful control, since inflow and outflow can increase the strict relative degree of the system (and hence the number of linearized variables which can be controlled).

Feedback linearization has been shown to be effective in exactly tracking thrombin trajectories in patients with both Factor-V Leiden and hæmophilia A, but only given the estimated rate constants of $\S 5$ and no constraints on the input. Adding constraints greatly degrades tracking. Now, an approach to address exactly this issue is (linear) model-predictive control: A model of the plant is used to "look ahead," each time step, a certain number $(N)$ of time steps into the future evolution of (the linearized part of) the system, from which the optimal input given the constraints is computed for all $N$ steps (this amounts to solving a quadratic programming problem). The look-ahead allows the controller to adjust present inputs in light of future constraints. We are 
currently implementing such a controller.

Finally, it must be demonstrated how the decomposition of the hybridsystem model of the entire clotting process (discussed in the introduction) can be used, in conjunction with these control techniques, to control clot formation. An approach based on reachability proofs on Petri nets will be demonstrated in a subsequent paper.

\section{Acknowledgments}

The authors would like to thank Drs. Shankar Sastry and Tom Budinger for their useful input; and especially Dr. Jerry Feldman for suggesting step-input control.

\section{References}

[1] Makin J, Narayanan S (2008) A hybrid-system model of human blood clotting. Technical report, International Computer Science Institute, 1947 Center Street; Berkeley, CA; 94703. URL http://www.icsi.berkeley.edu/ $\sim$ makin/BloodTR.pdf.

[2] Bungay S, Gentry P, Gentry R (2003) A mathematical model of lipid mediated thrombin generation. Mathematical Medicine and Biology 20:105129.

[3] Panteleev M, Zarnitsina V, Ataullakhanov F (2002) Tissue factor pathway inhibitor - a possible mechanism of action. European Journal of Biochemistry 269:2016-2031.

[4] Kogan A, Kardakov D, Khanin M (2001) Analysis of the activated partial thromboplastin time test using mathematical modeling. Thrombosis Research 101:299-310.

[5] Leipold R, Bozarth T, Racanelli A, Dicker I (1995) Mathematical model of serine protease inhibition in the tissue factor pathway to thrombin. The Journal ob Biological Chemistry 270:25383-25387.

[6] Qiao Y, Xu C, Zeng Y, Xu X, Zhao H, et al. (2004) The kinetic model and simulation of blood coagulation-the kinetic influence of activated protein c. Medical Engineering and Physics 26:341-347.

[7] Butenas S, Orfeo T, Gissel M, Brummel K, Mann K (2004) The significance of circulating factor ixa in blood. The Journal of Biological Chemistry 279:22875-22882.

[8] Pohl B, Beringer C, Bomhard M, Keller F (1994) The quick machine-a mathematical model for the extrinsic activation of coagulation. Hæmostasis 24:325-337. 
[9] Luan D, Zai M, Varner JD (2007) Computationally derived points of fragility of a human cascade are consistent with current therapeutic strategies. PLoS Computational Biology 3:1347-1359.

[10] Mitchell I, Bayen AM, Tomlin CJ (2001) Validating a Hamilton-Jacobi approximation to hybrid system reachable sets. In: Benedetto MDD, Sangiovanni-Vincentelli AL, editors, Hybrid Systems: Computation and Control, 4th International Workshop, HSCC 2001, Rome, Italy, March 2830, 2001, Proceedings. Springer, volume 2034 of Lecture Notes in Computer Science, pp. 418-432.

[11] Sastry SS (1999) Nonlinear Systems: Analysis, Stability, and Control. New York: Springer-Verlag.

[12] Bastin G, Lévine J (1993) On state accessibility in reaction systems. IEEE Transactions on Automatic Control 38:733-742.

[13] Henson MA, Seborg DE (1996) Feedback linearizing control. In: Seborg DE, Henson MA, editors, Nonlinear Process Control, Upper Saddle River, NJ: Prentice Hall. pp. 149-231.

[14] Olson ST (1988) Transient kinetics of heparin-catalyzed protease inactivation by antithrombin III. The Journal of Biological Chemistry 263:16981708 .

[15] Fersht A (1999) Structure and Mechanism in Protein Science. New York: W.H. Freeman and Company.

[16] Butenas S, van't Veer C, Mann KG (1999) 'Normal' thrombin generation. Blood 94:2169-2178.

[17] Brummel K, Paradis S, Butenas S, Mann K (2002) Thrombin functions during tissue factor-induced blood coagulation. Blood 100:148-152. 


\begin{tabular}{|c|c|c|c|}
\hline Reaction & $\mathrm{k}_{\mathrm{on}}\left(\mathrm{nM}^{-1} \mathrm{~s}^{-1}\right)$ & $\mathrm{k}_{\mathrm{off}}\left(\mathrm{s}^{-1}\right)$ & $\mathrm{k}_{\text {cat }}\left(\mathrm{s}^{-1}\right)$ \\
\hline $\mathrm{II}+\mathrm{LBS} \rightleftharpoons \mathrm{II}_{\mathrm{L}}$ & 0.0043 & 1 & - \\
\hline $\mathrm{mIIa}+\mathrm{LBS} \rightleftharpoons \mathrm{mIIa}_{\mathrm{L}}$ & 0.05 & 0.475 & - \\
\hline $\mathrm{V}+\mathrm{LBS} \rightleftharpoons \mathrm{V}_{\mathrm{L}}$ & 0.05 & 0.145 & - \\
\hline $\mathrm{Va}+\mathrm{LBS} \rightleftharpoons \mathrm{Va}_{\mathrm{L}}$ & 0.057 & 0.17 & - \\
\hline $\mathrm{VII}+\mathrm{LBS} \rightleftharpoons \mathrm{VII}_{\mathrm{L}}$ & 0.05 & 0.66 & - \\
\hline $\mathrm{VIIa}+\mathrm{LBS} \rightleftharpoons \mathrm{VIIa}_{\mathrm{L}}$ & 0.05 & 0.227 & - \\
\hline $\mathrm{VIII}+\mathrm{LBS} \rightleftharpoons \mathrm{VIII}_{\mathrm{L}}$ & 0.05 & 0.1 & - \\
\hline $\mathrm{VIIIa}+\mathrm{LBS} \rightleftharpoons \mathrm{VIIIa}_{\mathrm{L}}$ & 0.05 & 0.335 & - \\
\hline $\mathrm{IX}+\mathrm{LBS} \rightleftharpoons \mathrm{IX}_{\mathrm{L}}$ & 0.05 & 0.115 & - \\
\hline $\mathrm{IXa}+\mathrm{LBS} \rightleftharpoons \mathrm{IXa}_{\mathrm{L}}$ & 0.05 & 0.115 & - \\
\hline $\mathrm{X}+\mathrm{LBS} \rightleftharpoons \mathrm{X}_{\mathrm{L}}$ & 0.01 & 1.9 & - \\
\hline $\mathrm{Xa}+\mathrm{LBS} \rightleftharpoons \mathrm{Xa}_{\mathrm{L}}$ & 0.029 & 3.3 & - \\
\hline $\mathrm{APC}+\mathrm{LBS} \rightleftharpoons \mathrm{APC}_{\mathrm{L}}$ & 0.05 & 3.5 & - \\
\hline $\mathrm{PS}+\mathrm{LBS} \rightleftharpoons \mathrm{PS}_{\mathrm{L}}$ & 0.05 & 0.2 & - \\
\hline $\mathrm{VIIIa}_{\mathrm{i}}+\mathrm{LBS} \rightleftharpoons \mathrm{VIIIa}_{\mathrm{i}, \mathrm{L}}$ & 0.05 & 0.335 & - \\
\hline $\mathrm{Va}_{\mathrm{i}}+\mathrm{LBS} \rightleftharpoons \mathrm{VIIIa}_{\mathrm{i}, \mathrm{L}}$ & 0.057 & 0.17 & - \\
\hline $\mathrm{PC}+\mathrm{LBS} \rightleftharpoons \mathrm{PC}_{\mathrm{L}}$ & 0.05 & 11.5 & - \\
\hline $\mathrm{TF}_{\mathrm{L}}+\mathrm{VIIa}_{\mathrm{L}} \rightleftharpoons \mathrm{TF}: \mathrm{VIIa}_{\mathrm{L}}$ & 0.5 & 0.005 & - \\
\hline $\mathrm{TF}_{\mathrm{L}}+\mathrm{VII}_{\mathrm{L}} \rightleftharpoons \mathrm{TF}: \mathrm{VII}_{\mathrm{L}}$ & 0.005 & 0.005 & - \\
\hline 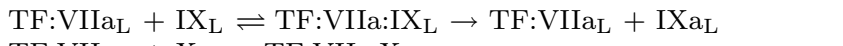 & 0.01 & 2.09 & 0.34 \\
\hline TF:VIIa ${ }_{L}+\mathrm{X}_{\mathrm{L}} \rightleftharpoons \mathrm{TF}:$ VIIa: $\mathrm{X}_{\mathrm{L}} \rightarrow$ & & & \\
\hline TF:VIIa:Xa $\mathrm{L}_{\mathrm{L}} \rightarrow \mathrm{TF}: \mathrm{VIIa}_{\mathrm{L}}+\mathrm{Xa}_{\mathrm{L}}$ & 0.1 & 32.5 & $1.5,1$ \\
\hline $\mathrm{TF}: \mathrm{VII}_{\mathrm{L}}+\mathrm{XaL} \rightleftharpoons \mathrm{TF}: \mathrm{VII}: \mathrm{Xa}_{\mathrm{L}} \rightarrow \mathrm{TF}: \mathrm{VII}_{\mathrm{L}}+\mathrm{Xa}_{\mathrm{L}}$ & 0.05 & 44.8 & 15.2 \\
\hline 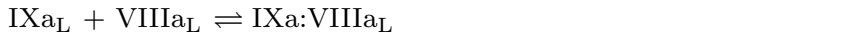 & 0.1 & 0.2 & - \\
\hline $\mathrm{Xa}_{\mathrm{L}}+\mathrm{Va}_{\mathrm{L}} \rightleftharpoons \mathrm{Xa}: \mathrm{Va}_{\mathrm{L}}$ & 1 & 1 & - \\
\hline IXa:VIIIa $_{L}+\mathrm{X}_{\mathrm{L}} \rightleftharpoons$ IXa:VIIIa:X $\mathrm{L} \rightarrow$ IXa:VIIIa $_{L}+\mathrm{Xa}_{\mathrm{L}}$ & 0.1 & 10.7 & 8.3 \\
\hline $\mathrm{V}_{\mathrm{L}}+\mathrm{Xa}_{\mathrm{L}} \rightleftharpoons \mathrm{V}: \mathrm{Xa}_{\mathrm{L}} \rightarrow \mathrm{Va}_{\mathrm{L}}+\mathrm{Xa}_{\mathrm{L}}$ & 0.1 & 1 & 0.043 \\
\hline $\mathrm{VIII}_{\mathrm{L}}+\mathrm{Xa}_{\mathrm{L}} \rightleftharpoons \mathrm{VIII}: \mathrm{Xa}_{\mathrm{L}} \rightarrow \mathrm{VIIIa}_{\mathrm{L}}+\mathrm{Xa}_{\mathrm{L}}$ & 0.1 & 2.1 & 0.023 \\
\hline $\mathrm{V}_{\mathrm{L}}+\mathrm{IIa} \rightleftharpoons \mathrm{V}: \mathrm{IIa}_{\mathrm{L}} \rightarrow \mathrm{Va}_{\mathrm{L}}+\mathrm{IIa}$ & 0.1 & 6.94 & 0.23 \\
\hline VIII $_{L}+\mathrm{IIa} \rightleftharpoons$ VIII:IIa $L$ & 0.1 & 13.8 & 0.9 \\
\hline $\mathrm{Xa}: \mathrm{Va}_{\mathrm{L}}+\mathrm{II}_{\mathrm{L}} \rightleftharpoons \mathrm{Xa}: \mathrm{Va} \mathrm{II}_{\mathrm{L}}$ & 0.1 & 100 & - \\
\hline $\mathrm{Xa}: \mathrm{Va}_{\mathrm{L}}+\mathrm{mIIa}_{\mathrm{L}} \rightleftharpoons \mathrm{Xa}: \mathrm{Va}_{\mathrm{mIIa}}$ & 0.1 & 66 & - \\
\hline Xa:Va:II $I_{L} \rightarrow$ Xa:Va:mIIa $L$ Xa:Va ${ }_{L}+$ IIa & 13 & 15 & - \\
\hline $\mathrm{VII}_{\mathrm{L}}+\mathrm{Xa}_{\mathrm{L}} \rightleftharpoons \mathrm{VII}: \mathrm{Xa}_{\mathrm{L}} \rightarrow \mathrm{VIIa}_{\mathrm{L}}+\mathrm{Xa}_{\mathrm{L}}$ & 0.05 & 44.8 & 15.2 \\
\hline $\mathrm{XI}+\mathrm{IIa} \rightleftharpoons \mathrm{XI}: \mathrm{IIa} \rightarrow \mathrm{XIa}+\mathrm{IIa}$ & 0.1 & 10 & 1.43 \\
\hline $\mathrm{APC}_{\mathrm{PS}} \mathrm{L}+\mathrm{VIIIa}_{\mathrm{L}} \rightleftharpoons \mathrm{APC}: \mathrm{PS}: \mathrm{VIIIa}_{\mathrm{L}} \rightarrow \mathrm{APC} \mathrm{PS}_{\mathrm{L}}+\mathrm{VIIIa}_{\mathrm{i}, \mathrm{L}}$ & 0.1 & 1.6 & 0.4 \\
\hline $\mathrm{APC}: \mathrm{PS}_{\mathrm{L}}+\mathrm{Va}_{\mathrm{L}} \rightleftharpoons \mathrm{APC}: \mathrm{PS}: \mathrm{Va}_{\mathrm{L}} \rightarrow \mathrm{APC}: \mathrm{PS}_{\mathrm{L}}+\mathrm{Va}_{\mathrm{i}, \mathrm{L}}$ & 0.1 & 1.6 & 0.4 \\
\hline $\mathrm{TFPI}+\mathrm{Xa} \rightleftharpoons \mathrm{TFPI}: \mathrm{Xa}$ & 0.016 & 0.000333 & - \\
\hline TFPI:Xa + TF:VIIaL $\rightleftharpoons$ TFPI:Xa:TF:VIIaL & 0.01 & 0.0011 & - \\
\hline $\mathrm{IXa}+\mathrm{AT} \rightarrow \mathrm{IXa}: \mathrm{AT}$ & $4.9 \times 10^{-7}$ & - & - \\
\hline $\mathrm{Xa}+\mathrm{AT} \rightarrow \mathrm{Xa}: \mathrm{AT}$ & $2.3 \times 10^{-6}$ & - & - \\
\hline $\mathrm{IIa}+\mathrm{AT} \rightarrow \mathrm{IIa}: \mathrm{AT}$ & $6.83 \times 10^{-5}$ & - & - \\
\hline $\mathrm{V}_{\mathrm{L}}+\mathrm{mIIa}_{\mathrm{L}} \rightleftharpoons \mathrm{V}: \mathrm{mIIa}_{\mathrm{L}} \rightarrow \mathrm{Va}_{\mathrm{L}}+\mathrm{mIIa}_{\mathrm{L}}$ & 0.1 & 6.94 & 1.035 \\
\hline 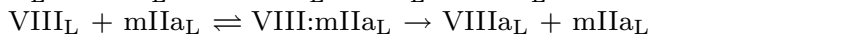 & 0.1 & 13.8 & 0.9 \\
\hline $\mathrm{IIa}+\mathrm{Tm}_{\mathrm{L}} \rightleftharpoons \mathrm{IIa}: \mathrm{Tm}_{\mathrm{L}}$ & 1 & 0.5 & - \\
\hline IIa:Tm $\mathrm{Tm}_{\mathrm{L}}+\mathrm{PC}_{\mathrm{L}} \rightleftharpoons \mathrm{IIa}: \mathrm{Tm}: \mathrm{PC}_{\mathrm{L}} \rightarrow \mathrm{IIa}_{\mathrm{Tm}}+\mathrm{APC}_{\mathrm{L}}$ & 0.1 & 6.4 & 3.6 \\
\hline $\mathrm{mIIa}+\mathrm{AT} \rightarrow$ mIIa:AT & $6.83 \times 10^{-6}$ & - & - \\
\hline $\mathrm{APC}_{\mathrm{L}}+\mathrm{PS}_{\mathrm{L}} \rightleftharpoons \mathrm{APC}: \mathrm{PS}_{\mathrm{L}}$ & 0.1 & 0.5 & - \\
\hline $\mathrm{XIa}+\mathrm{IX}_{\mathrm{L}} \rightleftharpoons \mathrm{XIa}: \mathrm{IX}_{\mathrm{L}} \rightarrow \mathrm{XIa}+\mathrm{IXa}_{\mathrm{L}}$ & 0.01 & 1.417 & 0.183 \\
\hline
\end{tabular}

Table 1: The chemical reactions of the critical subsystem modeled in this study. The equations and their rate constants were all drawn from [2]. 


\begin{tabular}{|c|c|c|}
\hline Reaction & $\mathrm{k}_{\mathrm{on}}\left(\mathrm{nM}^{-1} \mathrm{~s}^{-1}\right)$ & $\mathrm{k}_{\text {off }}\left(\mathrm{s}^{-1}\right)$ \\
\hline Hep + AT $\rightleftharpoons$ Hep:AT & 0.1 & 10 \\
\hline Hep + IIa $\rightleftharpoons$ Hep:IIa & 0.1 & 10 \\
\hline $\mathrm{AT}+\mathrm{IIa} \rightleftharpoons \mathrm{AT}: \mathrm{IIa}$ & 0.1 & 10 \\
\hline Hep:AT + IIa $\rightleftharpoons$ Hep:AT:IIa & 0.1 & 10 \\
\hline Hep:IIa + AT $\rightleftharpoons$ Hep:AT:IIa & 0.1 & 10 \\
\hline IIa:AT + AT $\rightleftharpoons$ Hep:AT:IIa & 0.1 & 10 \\
\hline IIa-AT + Hep $\rightleftharpoons$ Hep:AT:IIa & 0.1 & 10 \\
\hline
\end{tabular}

Table 2: The chemical reactions of the heparin reactions. The equations were drawn from [14] but the rate constants were estimated (see text).

\begin{tabular}{ll|ll|lc}
\hline Species & Conc. (nM) & Species & Conc. (nM) & Species & Conc. (nM) \\
\hline Tissue Factor & 0.005 & Factor VIII & 0.7 & TFPI & 2.5 \\
Factor II & 1400 & Factor IX & 90 & Antithrombin & 3400 \\
Factor V & 20 & Factor XI & 30 & Protein C & 60 \\
Factor VII & 10 & Factor X & 170 & Protein S & 300 \\
Factor VIIa & 0.1 & Thrombomodulin & 1 & LBS & 3396 \\
\hline
\end{tabular}

Table 3: Initial conditions 